\title{
Explicit Expressions for the Determinants of Certain Matrices
}

\author{
By J. L. Lavoie and R. Michaud
}

Abstract. Explicit expressions for the determinants of twenty particular $n$-square matrices are given.

1. Introduction. A few years ago one of the authors [3] obtained explicit expressions for the determinants of certain particular $n$-square matrices by either decomposing these matrices into triangular factors or by transforming them into matrices whose determinants are well known. These operations were effected mainly by pre- or postmultiplications of the given matrix by specially constructed triangular matrices, so that certain summation theorems from the theory of generalized hypergeometric functions could be utilized.

Some interest was aroused by this method at the time, and it was thought that a systematic use of the many well-known summation theorems from the theory of generalized hypergeometric functions would lead to the explicit evaluation of determinants of a very general class of matrices. Unfortunately this does not seem to be the case. A search for a few very general theorems concluded with a collection of particular results.

While it is true that the expressions obtained in certain cases are remarkable, it is also true that, to borrow a phrase from the preface of John Riordan's latest book [4], they "seem to lose whatever memorable character they have by the profusion that surrounds them." It must also be said that the forms of the results are unpredictable and there seems to be no obvious criterion for their classification and no limit to their number, since one known result usually leads to another.

We would like to record here the expressions for the determinants of twenty particular matrices. Unfortunately lack of space prevents us from giving the details of the straightforward but rather lengthy manipulations involved. For convenience we have listed three known results, numbers (2.3), (2.11) with $q=1$, and (2.20), and the interested reader may consult [3] for the details.

It should be observed that Burchnall wrote two papers [1], [2] on a method of evaluating determinants which is closely related to, yet somewhat different from ours. The expressions (2.2), (2.3), and (2.11) below appear in these papers along with more general results.

It is clear that the following results can be applied in a number of different situations, but in addition to this their very existence is certainly interesting.

2. Some Particular Matrices and Their Determinants. The following table lists some of the results we have obtained. As usual, $A=\left(a_{i j}\right), i, j=1, \cdots, n$ is an

Received June 25, 1969.

AMS Subject Classifications. Primary 1520, 3320; Secondary 3315.

Key Words and Phrases. Evaluation of certain determinants, generalized hypergeometric functions, gamma functions. 
$n$-square matrix whose typical element is $a_{i j}$, $\operatorname{det}(A)$ is the determinant of $A$, and $\Gamma(z)$ is the gamma function. The reader can easily decide, in each case, for which values of the arbitrary parameters $p$ and $q$ the matrix $A$ is singular.

$a_{i j}$

$$
\Gamma(p+i-j) \quad(-1)^{n(n-1) / 2} \prod_{k=1}^{n}(k-1) ! \Gamma(p+1-k)
$$

$$
\Gamma(p+i+j) \quad \prod_{k=1}^{n}(k-1) ! \Gamma(p+1+k)
$$

$$
\frac{1}{\Gamma(p+i+j)} \quad(-1)^{n(n-1) / 2} \prod_{k=1}^{n} \frac{(k-1) !}{\Gamma(p+n+k)}
$$

$$
\begin{gathered}
\Gamma(p+i+j-1) \Gamma(p+q+i-j-1) \\
\prod_{k=1}^{n} \frac{(k-1) ! \Gamma(p+k) \Gamma(q-k) \Gamma(p+q-k)}{\Gamma(q-2 k+1)} \\
\frac{1}{\Gamma(p+i+j) \Gamma(q-i+j)} \\
\prod_{k=1}^{n} \frac{(k-1) ! \Gamma(p-q+2 k)}{\Gamma(p+n+k) \Gamma(q+k-1) \Gamma(p-q+k+1)} \\
\frac{1}{\Gamma(p+i-j) \Gamma(q-i-j)} \quad \frac{(k-1) ! \Gamma(p-q+2 k)}{(-1)^{n(n-1) / 2} \prod_{k=1}^{n} \frac{1(p+k-1) \Gamma(q-k-1) \Gamma(p-q+k+1)}{\Gamma(k-1)}}
\end{gathered}
$$




$$
\frac{\Gamma(q+i+j-1)}{\Gamma(p+i+j)}
$$

$$
\frac{1}{\{\Gamma(p-q+1)\}^{n}} \prod_{k=1}^{n} \frac{(k-1) ! \Gamma(q+k) \Gamma(p-q+k)}{\Gamma(p+n+k)}
$$

$$
\frac{\Gamma(q+i-j-1)}{\Gamma(p+i-j)}
$$

$$
\frac{(-1)^{n(n-1) / 2}}{\{\Gamma(p-q+1)\}^{n}} \prod_{k=1}^{n} \frac{(k-1) ! \Gamma(q-k) \Gamma(p-q+k)}{\Gamma(p+k-1)}
$$

$$
\frac{\Gamma(q+i-j-1)}{\Gamma(p+i+j)}
$$

$$
(-1)^{n(n-1) / 2} \prod_{k=1}^{n} \frac{(k-1) ! \Gamma(q-k) \Gamma(p+q+2 k-2)}{\Gamma(p+n+k) \Gamma(p+q+k-1)}
$$

(2.14) $\quad \frac{\Gamma(q+i+j-1)}{\Gamma(p+i-j)} \quad \prod_{k=1}^{n} \frac{(k-1) ! \Gamma(q+k) \Gamma(p+q+2 k-2)}{\Gamma(p+k-1) \Gamma(p+q+k-1)}$

(2.15) $\frac{\Gamma(q+i+2 j)}{\Gamma(p+q+i+j)} \quad \prod_{k=1}^{n} \frac{(k-1) ! \Gamma(q+2 k+1) \Gamma(2 p+q+2 k-2)}{\Gamma(p+q+n+k) \Gamma(2 p+q+k-1)}$

$$
\frac{\Gamma(p+2 i-j-1)}{\Gamma(p+i-j)} \quad(-1)^{n(n-1) / 2} \prod_{k=1}^{n}(k-1) !
$$

$$
\frac{\Gamma(q+i-2 j)}{\Gamma(p+q+i-j)}
$$

$$
(-1)^{n(n-1) / 2} \prod_{k=1}^{n} \frac{(k-1) ! \Gamma(q-2 k+1) \Gamma(2 p+q+2 k-2)}{\Gamma(p+q+k-1) \Gamma(2 p+q+k-1)}
$$

$$
\frac{\Gamma(p-i+j)}{\Gamma(p-2 i+j+1)} \quad \prod_{k=1}^{n}(k-1) !
$$




$$
\begin{gathered}
\frac{1}{\Gamma(q+i-j) \Gamma(p-i+j)} \\
\frac{1}{\{\Gamma(p+q-1)\}^{n}} \prod_{k=1}^{n} \frac{(k-1) ! \Gamma(p+q+k-2)}{\Gamma(p+k-1) \Gamma(q+k-1)} \\
\frac{1}{\Gamma\left(\frac{p+i+j+1}{2}\right) \Gamma\left(\frac{p-i+j+2}{2}\right)} \quad\left(\frac{2^{p+1}}{\sqrt{ } \pi}\right)^{n} \prod_{k=1}^{n} \frac{(2 k-2) !}{\Gamma(p+n+k)}
\end{gathered}
$$

Université Laval

Québec 10

Canada

1. J. L. Burchnall, "Some determinants with hypergeometric elements," Quart. J. Math. Oxford Ser., (2) v. 3, 1952, pp. 151-157. MR 14, 44.

2. J. L. BuRChNALL, "A method of evaluating certain determinants," Proc. Edinburgh Math. Soc., (2) v. 9, 1954, pp. 100-104. MR 16, 557.

3. J. L. Lavore, "On the evaluation of certain determinants," Math. Comp., v. 18, 1964, pp. 653-659. MR $30 \# 703$.

4. John RIORDAN, Combinatorial Identities, Wiley, New York, 1968. MR 38 \#53. 